\title{
Raucherentwöhnung bei Krankenhausmitarbeitern
}

\author{
Eine kontrollierte Interventionsstudie
}
A. Michalsen ${ }^{1}$
B. Richarz ${ }^{1}$
H. Reichart ${ }^{2}$
G. Spahn ${ }^{1}$
N. Konietzko ${ }^{3}$
G. J. Dobos ${ }^{1}$

\section{Smoking cessation for hospital staff. A controlled trial}

Hintergrund und Fragestellung: Die Raucherprävalenz in Deutschland ist unverändert hoch. Insbesondere unter Mitarbeitern in Krankenhäusern findet sich ein hoher Raucheranteil. Zur Umsetzung des „Rauchfreien Krankenhauses“ im Sinne einer Vorbildfunktion ist die Minimierung der Raucherprävalenz von Krankenhausmitarbeitern dringend notwendig. In dieser kontrollierten Studie wurde eine forcierte gemeinschaftliche Aktion „Rauchfreies Krankenhaus“ an einem Akutkrankenhaus durchgeführt.

Patienten und Methodik: Der Raucherstatus wurde initial mittels Selbsterhebungsbögen erfasst. Für Raucher wurde ein Entwöhnungsprogramm angeboten; dieses umfasste für alle Teilnehmer eine Beratung und kognitive Kurzintervention sowie die medikamentöse Nikotinersatztherapie mit Pflaster/Kaugummi. Teilnehmer in Gruppe B nahmen zusätzlich und freiwillig an einem strukturierten Stressreduktionsprogramm („Mindfulness based stress reduction“) in kleinen Gruppen über 20 Stunden teil, Teilnehmer in Gruppe A nicht. Primäres Zielkriterium war die kontinuierliche Abstinenz nach 3 Monaten, sekundäre Zielkriterien die Abstinenz nach 6 Wochen und 6 Monaten, der Verlauf der exspiratorischen CO-Konzentration und die Rauchintensität bei Rückfälligen.

Ergebnisse: Bei einer Rücklaufquote von $86 \%$ fanden sich in der Erhebung unter antwortenden 296 Mitarbeitern 140 Raucher (47,3\%), von denen 116 an der Entwöhnung teilnahmen (Gruppe A: n=63, Gruppe B: $n=53$ ). Insgesamt waren nach 3 und 6 Monaten 38,2\% und $29,7 \%$ aller teilnehmenden Mitarbeiter abstinent. In Gruppe B fanden sich höhere, jedoch nicht signifikant erhöhte Abstinenzraten an den Messzeitpunkten (3-Monatsabstinenz: A: 35\% vs. B: 42\%; 6-Monatsabstinenz: A: $20 \%$ vs. B: $41,2 \%$ ) sowie eine deutlichere Senkung der exspiratorischen CO-Konzentration und ausgeprägtere Reduktion der Anzahl gerauchter Zigaretten bei Rückfälligen.

Folgerung: Durch gemeinschaftliche und forcierte innerbetriebliche Aktionen mit ärztlich geleiteter Nikotinentwöhnungstherapie kann der hohe Anteil rauchender Krankenhausmitarbeiter relevant reduziert werden. Eine breitere Durchführung solcher Initiativen ist wünschenswert. Der Nutzen von begleitenden Stressreduktionsprogrammen bleibt in weiteren Studien zu überprüfen.
Background and objective: Smoking prevalence in Germany remains high instead of public information and education. Smoke free hospitals are propagated, but frequently the smoking prevalence in hospital staff is even higher than in the general population. The objectives of the study were to determine the prevalence of smoking amongst hospital employees in a german teaching hospital and subsequently to promote and optimise smoking cessation within controlled trial with additional evaluation of mindfulness based stress reduction, supported by a common workplace action.

Patients and methods: A hospital survey was carried out by anonymous questionnaires. Participation in a physician-supervised smoking cessation program with nicotine replacement, brief intervention and, additionally, in a 20-hour stress reduction program over 8 weeks (group B) was offered to all smokers. Primary outcomes were abstinence rates at 3 months, secondary endpoints included abstinence rates at 6 months, cigarette consumption in smokers and expiratory $\mathrm{CO}$-concentration.

Results: Out of 345 employees 296 (86\%) revealed their smoking status, 140 were smokers (47,3\%). 116 of 140 smokers agreed with participation in a smoking cessation program. 53 subjects participated additionally in the stress reduction groups (Group B), 63 subjects wished no additional stress reduction (Group A). Abstinence rates at 3 and 6 months were 38,2\% and $29,7 \%$ for all subjects. Participants with stress reduction showed non-significant higher abstinence rates ( 3 months: A: $35 \%$ vs. B: $42 \%$ and 6 months: A: $20 \%$ vs. B: $41,2 \%$ ), a significant lower COconcentration and a lower smoking intensity in recurrent smokers.

Conclusion: With a common work place action a substantial part of employees quits smoking. Thus, the study provides support for the active promotion of physician-supervided smoking cessation programs among hospital employees. Supportive mindfulness-based stress reduction may enhance smoking cessation, yet has to be further tested in larger randomised controlled trials.

${ }^{1}$ Abt. für Innere Medizin V und Integrative Medizin, Kliniken Essen-Mitte

${ }^{2}$ Abt. für Innere Medizin II, Pneumologie, Kliniken Essen-Mitte

${ }^{3}$ Ruhrlandklinik, Abt. Pneumologie-Universitätsklinikum Essen 
Nach Prognosen der Global Burden of Disease Study wird die Tabakabhängigkeit in den nächsten Jahrzehnen weltweit die häufigste Einzelursache für Krankheiten und vorzeitiges Sterben sein (23). In Deutschland sterben jährlich vermutlich zwischen 90000 und 140000 Menschen an den Folgen des Tabakkonsums (28). Während es inzwischen in den USA gelang, mit Unterstützung einer entsprechenden Gesetzgebung den Anteil an Rauchern in der Bevölkerung kontinuierlich zu senken, ist eine solche Entwicklung in Deutschland nicht erkennbar. Zwar ist hier der Anteil bei den Männern zuletzt leicht gesunken, bei den Frauen hingegen angestiegen. 1998 waren 37\% der Männer und $28 \%$ der Frauen Raucher (16).

Neben der primären Prävention muss es daher eines der obersten Ziele sein, möglichst viele Raucher zu einer erfolgreichen Entwöhnung zu bringen. Krankenhäuser sollten hierbei modellhaft wirken. Aus amerikanischen Studien ist bekannt, dass etwa 3/4 aller Raucher jedes Jahr Einrichtungen des Gesundheitswesens aufsuchen und etwa 70\% mit dem Rauchen aufhören wollen (25). Jedoch findet sich bei Mitarbeitern von Krankenhäusern häufig eine höhere Raucherprävalenz als im Bevölkerungsdurchschnitt $(5,12,14,24,33)$. Traditionell sind hierunter Berufsgruppen mit hohem Raucheranteil, wie z. B. die Krankenpflege $(22,27)$, vermutlich fördern typische Merkmale des modernen Krankenhausarbeitsplatzes wie Schichtdienst, hohes Verantwortungsprofil und Leistungsanforderung den arbeitsbezogenen Stress und damit den Nikotinkonsum. In einer 1992 publizierten deutschen Klinikerhebung fand sich eine Raucherprävalenz von 51\% bei Mitarbeitern der Krankenpflege und von $35 \%$ bei Klinikärzten (32).

Gesunderhaltung der Mitarbeiter und Vorbildfunktion des Krankenhauses sind klare Motive, um dem hohen Raucheranteil im deutschen Krankenhauspersonal entgegenzuwirken. Darüber hinaus sind rauchende Mitarbeiter nur in geringerem Maße für die aktive therapeutische Raucherentwöhnung von Patienten aktivierbar (22).

Ein spontaner Entwöhnungsversuch ohne Nikotinersatz und ohne akute Erkrankungssituation geht in nur ca. 5\% der Fälle in eine dauerhafte Abstinenz über (3,34). Inzwischen ist zur Raucherentwöhnung die medikamentöse Nikotinersatztherapie (NET) etabliert. Bei nicht akut erkrankten sind nach einem Jahr Abstinenzraten zwischen 10 und $20 \%$ zu erzielen $(2,6,20)$. Intensivierte Begleitmaßnahmen in Form von qualifizierter Beratung unter Einbeziehen verhaltenszentrierter Techniken können den langfristigen Entwöhnungserfolg weiter steigern, allerdings nur selten über 25\% (9). Höhere Abstinenzraten sind zumeist nur in vulnerablen Situationen, wie nach erstem Myokardinfarkt, erreichbar $(8,31)$. Für verhaltenstherapeutische Interventionen und Entspannungsverfahren ist ein zusätzlicher therapeutischer Nutzen nachgewiesen $(7,21)$, jedoch erschweren Methodenvielfalt und unterschiedliche Intensität dieser Verfahren eine zusammenfassende Bewertung.

Seit 1999 propagiert die Bundeszentrale für gesundheitliche Aufklärung in Deutschland die Aktion „Rauchfreies Krankenhaus“ als einen Teil des WHO-Projektes „Partnerschaftsprojekt Tabakabhängigkeit 1999-2001“ (35). Wir führten eine intensivierte und gemeinschaftliche innerbetriebliche Aktion zur Rau- cherentwöhnung bei allen Krankenhausmitarbeitern durch. Eine möglichst hohe Abstinenzrate sollte durch eine optimierte Beratung und Pharmakotherapie nach publizierten Richtlinien $(11,35)$ und durch die motivationsfördernde Wirkung der gemeinschaftlichen Aktion erreicht werden. Der Nutzen einer freiwilligen Teilnahme an einem supportiven Stressreduktionsprogramm sollte darüber hinaus in dieser Studie geprüft werden.

\section{Probanden und Methodilk}

Die Studie wurde am Knappschafts-Krankenhaus der Kliniken Essen-Mitte, gesundheitsförderndes Krankenhaus der WHO und akademisches Lehrkrankenhaus der Universität Essen, durchgeführt. Der Standort verfügt über insgesamt 270 vollstationäre Betten.

\section{Erhebung der Raucherprävalenz}

Mit Einverständnis der Mitarbeitervertretung und der Klinikleitung wurden zunächst alle Mitarbeiter des Knappschaftskrankenhauses der Kliniken Essen-Mitte mit einem hausinternen Brief über die geplante Studieninitiative informiert und zu ihrem Rauchverhalten mittels eines anonymisierten Antwortbogens befragt. Neben dem Raucherstatus wurden Geschlecht, Berufsgruppe und Lebensalter abgefragt. Im Rahmen eines nachfolgenden Aktionstages „Rauchfreies Krankenhaus“ wurden die Mitarbeiter über die Möglichkeit einer folgenden kostenfreien und medikamentös begleiteten Raucherentwöhnung im Rahmen einer wissenschaftlichen Studie unterrichtet.

\section{Raucherentwöhnungsstudie}

Die Studie wurde als kontrollierte, nicht randomisierte Behandlungsstudie konzipiert. Einschlusskriterien waren die Bereitschaft, das Rauchen aufzugeben und an der Studie teilzunehmen, Alter $>18$ Jahre, Rauchen von mehr als 10 Zigaretten täglich, Rauchgewohnheit seit mindestens 3 Jahren. Bekannte Gegenanzeigen der NET und die außerstudienmäßige Anwendung anderer Maßnahmen zur Tabakentwöhnung bildeten die Ausschlusskriterien. Das positive Votum der zuständigen Ethikkommission zur Studie lag vor.

\section{Nikotinentwöhnungsverfahren}

Alle Teilnehmer (Gruppe $A+B$ ) erhielten standardisiert eine begleitende kurze edukative und kognitive Therapie sowie eine kostenfreie medikamentöse NET durch eine in der Raucherentwöhnung speziell geschulte Studienärztin. Die weitere Begleittherapie umfasste eine Informationsgebung in Gruppen, eine Einzelberatung zur Nikotinentwöhnung mit ausführlicher Anamnese zum Rauchverhalten und Vergegenwärtigung der Gründe, nicht mehr zu rauchen, die antizipierende Bearbeitung möglicher Rückfallsituationen sowie konsekutiv die schriftliche Vereinbarung eines Raucher-Stopp-Tages. Weiter wurde eine persönliche oder telefonische Notfallberatung bereitgehalten. Nach Erfassung des Abhängigkeitsprofiles nach Fagerstrøm (13) sowie dem früher erfahrenen Auftreten von Entzugssymptomen wurde die NET verordnet und ausgeteilt. Leicht abhängigen Rauchern (Fagerstrøm 0-3 Punkte) wurde Membranpflaster Nicorette ${ }^{\circledR}(24,9 \mathrm{mg}$ über 4 Wochen, 16,6mg über 8 Wochen) und Nikotinkaugummi Nicorette ${ }^{\circledR} 2 \mathrm{mg}$ ad libitum angeboten. Mittel und stark abhän- 
gige Raucher (Fagerstrøm 4-10 Punkte) erhielten bei gleicher Basistherapie zusätzlich Nikotinkaugummi Nicorette ${ }^{\circledR}$ $4 \mathrm{mg}$. Nicorette ${ }^{\circledR}$ Nasalspray $10 \mathrm{mg} / \mathrm{ml}$ wurde als Notfallmedikation zur Verfügung gestellt.

\section{Stressreduktionsprogramm}

Während der Erstinformation wurden alle Teilnehmer auf die Möglichkeit der Teilnahme an einem zusätzlichen Stress-Reduktionsprogramm aufgeklärt. Hierbei wurde der zeitliche Mehraufwand dieses Programmes betont. Die endgültige $\mathrm{Zu}-$ ordnung zur Stressreduktion (Gruppe B) erfolgte, wenn der Teilnehmer die Bereitschaft zur regelmäßigen Teilnahme an den Schulungsprogrammen sowie täglich 30 Minuten häuslichen Übungen zeigte. Das Programm umfasste über 8 Wochen einmal wöchentlich 2,5-stündige Schulungen durch einen ausgebildeten Therapeuten in kleinen Gruppen mit 10-20 Teilnehmern. Die vermittelte Stressreduktionstechnik basierte auf dem Programm der Stress-Reduction Clinic der Massachusetts-University und dem Konzept der „mindfulness based stress reduction“ $(17,18)$. Die Schulungstermine umfassten theoretische Inhalte der Stressphysiologie, Übungen zu spezifischen Entspannungstechniken, Atemübungen und Yoga. Darüber hinaus wurde die so genannte „Achtsamkeit“ als nicht bewertendes selbstreflexives Wahrnehmen von Reaktionsmustern vermittelt.

\section{Messmethoden}

Die initiale Befundaufnahme umfasste Body-Mass-Index und Puls/ Blutdruck nach 5-minütiger Ruhe. Die Zielparameter wurden nach 6 Wochen telefonisch sowie nach 3 und 6 Monaten durch persönliches Interview erfasst, bei Nicht-Einhalten des Termines durch Telefongespräch und nachfolgend zeitnahes persönliches Interview. Die Messung der Kohlenmonoxid (Co-) Konzentration (Gerät „Smokerlyzer“, Fa. Bedfont, Köln) im Exspirium erfolgte bei Studienbeginn sowie nach 3 und 6 Monaten. Zur Kategorisierung einer Abstinenz musste eine Kohlenmonoxidmessung vorliegen.

Primäres Zielkriterium der Studie war die Abstinenzrate nach 3 Monaten. Hierbei wurde von kontinuierlicher Abstinenz ausgegangen. Sekundäre Zielkriterien waren die Abstinenzrate nach 6 Wochen und nach 6 Monaten, die CO-Konzentration im Expirium nach 3 und 6 Monaten und das Ausmaß der Gewichtszunahme nach 6 Monaten. Die Anzahl der gerauchten Zigaretten wurde für alle nicht kontinuierlich Abstinenten nach 3 und 6 Monaten zum Ausgangswert verglichen und daraus die mittlere Reduktion des Rauchkonsums im Bezugskollektiv der verbleibenden Raucher errechnet. Die Veränderungen der untersuchten Parameter innerhalb der Behandlungsgruppen wurden mittels Vorzeichen-Rang-Test (Wilcoxon signed rank test) geprüft. Für den Vergleich der beiden Behandlungsarme wurde der Wilcoxon-Rangsummentest für unverbundene Stichproben eingesetzt. Für 2x2 Kontingenztafeln wurde der exakte Test nach Fischer verwendet. Zur Erkennung ungleich verteilter Variablen zwischen den Behandlungarmen wurde der Einfluss der Kovariablen Geschlecht, Alter, Berufsgruppe, Länge der Raucheranamnese und Fagerstrøm-Score in ein Regressionsmodell in Bezug auf die 3- und 6- Monatsabstinenz einbezogen.

Für die Fallzahlschätzung wurde bei 345 Mitarbeitern nach internen Schätzungen ein Raucheranteil von 50\% erwartet. Auf- grund der gemeinschaftlichen Aktivität mit hoher Zielidentifikation sowie der Einbindung der Mitarbeitervertretung und der Geschäftsführung wurde von einem hohen Anteil von etwa 50\% Entwöhnungswilligen in der Vorbefragung ausgegangen. Es wurde eine Abstinenzrate nach 3 Monaten von 15\% in der konventionellen Gruppe und 35\% in der additiv behandelten Gruppe erwartet. Die Wahrscheinlichkeit einen signifikanten Unterschied zwischen den Gruppen aufzuzeigen (Power) bei zweiseitiger Hypothesentestung betrug $80 \%$ bei einer Gruppengröße von je 50 Teilnehmern. Die Schätzung der Effektstärke der Stress-Intervention beruhte auf katamnestischen Daten zur Raucherentwöhnung der Massachusetts-Klinik.

\section{Ergebnisse}

\section{Erhebung der Raucherprävalenz}

Von insgesamt 345 Beschäftigten am Knappschaftskrankenhaus der Kliniken Essen-Mitte antworteten 296 in der initialen anonymen Befragung zum Raucherstatus, die Antwortquote lag damit bei $86 \%$. Bei einem Durchschnittsalter von 38 Jahren bezeichneten sich 140 Mitarbeiter (47,3\%) aktuell als Raucher, 156 als Nichtraucher (52,7\%). Raucher konsumierten im Mittel 25,9 \pm 10,3 Zigaretten täglich. Von 138 Mitarbeitern der Krankenpflege waren 79 Raucher (57,2\%), hierbei 24 von 26 der männlichen Mitarbeiter in der Krankenpflege (92,3\%). Im ärztlichen Personal fanden sich neun Raucher bei einem Rücklauf von 38 Fragebögen (23,6\% Raucher). Unter insgesamt 60 männlichen und 236 weiblichen Mitarbeitern war der Raucheranteil prozentual bei Männern höher (63,3\% vs. 43,2\%). Von den 140 Rauchern waren 116 (83\%) zur sofortigen Raucherentwöhnung motiviert und mit der Studienteilnahme einverstanden. 24 Raucher (17\%) hatten kein Interesse an einer Raucherentwöhnung. Unter den zum Raucherstopp motivierten Mitarbeitern wünschten 53 Personen (45\% der Entwöhnungswilligen) die Teilnahme am Stressreduktionsprogramm (Gruppe B).

\section{Raucherentwöhnung}

Das mittlere Alter lag in den Behandlungsgruppen bei 37 (A) und 41 Jahren (B). In Gruppe B überwog der Anteil der weiblichen Teilnehmer deutlich den der männlichen. Mitarbeiter der Krankenpflege waren in beiden Gruppen am häufigsten vertreten. An beiden Gruppen nahmen jeweils zwei Ärzte teil. Die Ausgangsdaten in beiden Gruppen sind in Tab.1 wiedergegeben, die Daten zum Rauchverhalten in Tab.2. Es fanden sich hierbei keine signifikanten Gruppenunterschiede. 80\% der Teilnehmer hatten bereits vor dem 20. Lebensjahr zu rauchen begonnen, dementsprechend lag die mittlere Dauer der Raucheranamnese bei etwa 20 Jahren. Die Teilnehmer rauchten im Mittel etwa 25 Zigaretten täglich. Mehr als $90 \%$ hatten schon einen oder mehrere Entzugsversuche hinter sich, davon in etwa 30\% mit Nikotinersatz. In der überwiegenden Zahl der Fälle war die frühere NET ohne ärztliche Anleitung erfolgt. Mehr als die Hälfte der Teilnehmer gab an, eine mit der Nikotinentwöhnung verbundene Gewichtszunahme zu befürchten.

In Gruppe A konnten die Daten von 60 der 63 Teilnehmer jeweils an den drei Messzeitpunkten komplett erhoben werden, in Gruppe B von 50 (6 Wochen, 3 Monate) bzw. 51 von 53 (6 Monate) Teilnehmern. Nach 3 Monaten waren in Gruppe A 21 
Tab.1 Ausgangsdaten in der Gesamtpopulation und in beiden Gruppen. BMI = Body- Mass-Index.

\begin{tabular}{llll}
\hline & $\begin{array}{l}\text { Gruppe } \boldsymbol{A} \\
(\mathbf{n = 6 3 )}\end{array}$ & $\begin{array}{l}\text { Gruppe B } \\
(\mathbf{n = 5 3 )}\end{array}$ & $\begin{array}{l}\text { Gesamtpopulation } \\
(\mathbf{n = 1 1 6 )}\end{array}$ \\
\hline Alter (Jahre) & $37,2 \pm 10,7$ & $40,6 \pm 9,7$ & $39,0 \pm 10,2$ \\
BMI (kg/m²) & $25,2 \pm 4,3$ & $24,2 \pm 4,5$ & $24,7 \pm 4,3$ \\
Weibl. Geschlecht (\%) & 54,0 & 73,6 & 63,0 \\
Beruf(\%): & & & \\
Pflege & 43,5 & 28,9 & 35,9 \\
Arzt/Ärztin & 3,2 & 3,7 & 3,4 \\
Sonstige & 53,3 & 67,4 & 60,7 \\
$\begin{array}{l}\text { Feste Partnerschaft (\%) } \\
\text { Rauchender Partner (\%) }\end{array}$ & 85,7 & 82,7 & 84,2 \\
& 54,0 & 47,1 & 50,9 \\
\hline
\end{tabular}

Tab.2 Rauchverhalten und Raucheranamnese vor Studienbeginn.

\begin{tabular}{|c|c|c|c|}
\hline & $\begin{array}{l}\text { Gruppe A } \\
(n=63)\end{array}$ & $\begin{array}{l}\text { Gruppe B } \\
(n=53)\end{array}$ & Gesamt \\
\hline Dauer des Rauchens (Jahre) & $18,6 \pm 9,3$ & $21,1 \pm 8,5$ & $19,8 \pm 8,9$ \\
\hline Beginn bis 20. Lebensjahr (\%) & 81,5 & 94,2 & 87,2 \\
\hline $\begin{array}{l}\text { Entwöhnungsversuche (\%) } \\
\text { noch nie } \\
\text { einmal } \\
2-5 \mathrm{mal} \\
>5 \mathrm{mal} \\
\text { mit Nikotinersatz }\end{array}$ & $\begin{array}{l}7,4 \\
42,6 \\
44,4 \\
5,6 \\
17,5\end{array}$ & $\begin{array}{l}8,0 \\
44,0 \\
40,0 \\
4,0 \\
41,5\end{array}$ & $\begin{array}{l}7,7 \\
43,3 \\
42,3 \\
4,7 \\
28,4\end{array}$ \\
\hline $\begin{array}{l}\text { Anzahl gerauchter } \\
\text { Zigaretten/Tag }\end{array}$ & $24,5 \pm 10,5$ & $26,4 \pm 10,8$ & $25,6 \pm 10,7$ \\
\hline $\begin{array}{l}\text { Angst vor Gewichtszunahme } \\
\text { bei Raucherstopp (\%) }\end{array}$ & 57,1 & 58,5 & 57,8 \\
\hline Fagerstrøm-Score: & $4,6 \pm 2,3$ & $5,2 \pm 2,2$ & $4,9 \pm 2,3$ \\
\hline $0-3(\%)$ & 42,8 & 28,3 & 36,2 \\
\hline $4-7(\%)$ & 41,4 & 60,3 & 50,0 \\
\hline $8-10(\%)$ & 15,8 & 11,3 & 13,8 \\
\hline
\end{tabular}

Teilnehmer (35\%) abstinent, weitere 15 (25\%) gaben eine Reduktion ihres Zigarettenkonsums an. In Gruppe B waren nach 3 Monaten 21 Teilnehmer (42\%) abstinent, weitere 12 (24\%) hatten den Zigarettenkonsum reduziert. Nach 6 Monaten waren die Unterschiede zwischen den beiden Behandlungsgruppen ausgeprägter mit noch 12 abstinenten Teilnehmern (20\%) in Gruppe A und $21(42 \%)$ in Gruppe B. Unter den rückfälligen Rauchern hatten in Gruppe A 21 (35\%) den Zigarettenkonsum reduziert, in Gruppe B 12 (23,5\%). Die Gruppenunterschiede der Abstinenz nach 3 und 6 Monaten erreichten jedoch nicht das Signifikanzniveau ( 3 Monate: $p=0,87 ; 6$ Monate: $p=0,22$ ). Die Abstinenzraten sind in Tab. 3 zusammengefasst.

In der Regressionsanalyse konnte kein signifikanter Einfluss der Kovariablen Alter, Geschlecht, Berufsgruppe, Länge der Raucheranamnese und Fagerstrøm-Score auf die Abstinenzrate festgestellt werden.

Die Reduktion des Rauchkonsums bei rückfälligen Rauchern führte zu einer mittleren Anzahl von im Mittel 10 vs. 4 gerauchten Zigaretten in Gruppe A vs. B nach 6 Monaten. Die Reduktion war damit ausgeprägter in der Behandlungsgruppe $B(p=0,01)$. Die Messungen der CO-Konzentration konnten komplett (Be-
Tab.3 Absolute und prozentuale Abstinenzraten (kontinuierliche Abstinenz) zu den 3 Messzeitpunkten 6 Wochen (tel.), 3 Monate, 6 Monate in beiden Gruppen und in der Gesamtpopulation. Es wurden jeweils 60 Teilnehmer in Gruppe A und 50 bzw. 51 Teilnehmer in Gruppe B zu den 3 Messzeitpunkten erfasst.

\begin{tabular}{llll}
\hline & $\begin{array}{l}\text { Abstinenz } \\
\mathbf{6} \text { Wochen } \\
\text { A: } n=60 \\
\text { B: } n=50\end{array}$ & $\begin{array}{l}\text { Abstinenz } \\
\text { 3 Monate } \\
\text { A: } n=60 \\
\text { B: } n=50\end{array}$ & $\begin{array}{l}\text { Abstinenz } \\
\mathbf{6} \text { Monate } \\
\text { A: } n=60 \\
\text { B: } n=51\end{array}$ \\
\hline Gruppe A & $30(50,0 \%)$ & $21(35,0 \%)$ & $12(20,0 \%)$ \\
Gruppe B & $37(74,0 \%)$ & $21(42,0 \%)$ & $21(41,2 \%)$ \\
Gesamt & $67(60,9 \%)$ & $42(38,2 \%)$ & $33(29,7 \%)$ \\
\hline
\end{tabular}

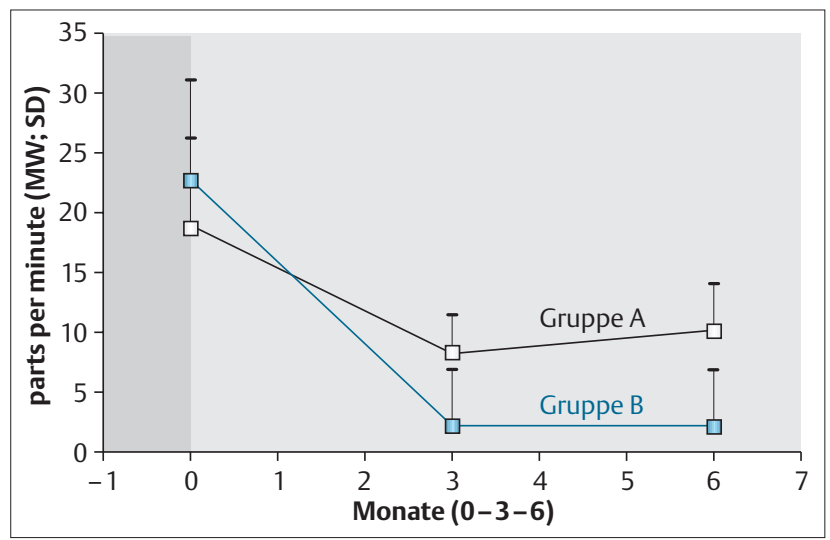

Abb.1 CO-Konzentration (parts per minute) im Exspirium (Mittelwert, SD) bei Beginn (Monat 0) sowie nach 3 und 6 Monaten in Gruppen $A(n=38), B(n=32)$. Signifikante Reduktion nach 3 und 6 Monaten in beiden Gruppen $(p<0,001)$. $P=0,03$ für Gruppenunterschied nach 6 Monaten.

ginn, 3 und 6 Monate) bei 70 Teilnehmern (38 in Gruppe A und 32 in Gruppe B) durchgeführt werden, darunter alle Teilnehmer, die sich als abstinent bezeichneten (Abb.1). In beiden Behandlungsgruppen war eine signifikante Reduktion der mittleren CO-Werte festzustellen $(\mathrm{p}<0,001)$, die Reduktion nach 6 Monaten war in Gruppe B ausgeprägter $(p=0,03)$.

Nikotinersatzpräparate waren nahezu allen Patienten bei Aufnahme verordnet worden. Nach 6 Monaten setzten 8 Teilnehmer in Gruppe A und 7 Teilnehmer in Gruppe B noch Nikotinersatzpräparate ein. Jeweils 20 Patienten (31,7\% und 37,7\%) gaben im Verlauf Beschwerden an, die ihrer Meinung nach in Zusammenhang mit der Anwendung ihres Nikotinersatzpräparates standen. Hierbei wurden am häufigsten Geschmacksstörungen (Kaugummi) und Allergien (Pflaster) registriert. Nikotinspray als Notfallmedikament wurde nicht in Anspruch genommen. Das mittlere Körpergewicht erhöhte sich in beiden Behandlungsgruppen signifikant mit im Mittel 2,4kg in Gruppe A und $1,7 \mathrm{~kg}$ in Gruppe B ( $<<0,001$, jeweils). Die Unterschiede der Gewichtszunahme zwischen den Gruppen waren nicht signifikant. 


\section{Diskussion}

In der vorliegenden Studie wurde im Rahmen einer gemeinschaftlichen Aktion in einem Krankenhaus der Regelversorgung die Raucherprävalenz der Mitarbeiter erhoben und nachfolgend ein strukturiertes Raucherentwöhnungsprogramm durchgeführt.

In der initialen Erhebung konnte eine hohe Antwortquote von $86 \%$ erzielt werden. Der dabei gefundene hohe Raucheranteil von über $47 \%$ bestätigte die subjektive Einschätzung, dass sich unter Krankenhausmitarbeitern eine alarmierend hohe Raucherprävalenz findet. Auch in einer früheren Erhebung fanden Troschke et al. 1992 eine ähnlich hohe Raucherprävalenz bei deutschem Klinikpersonal (32). Ein Rückgang des Gesamtanteiles rauchender Mitarbeiter scheint nicht stattzufinden, vermutlich auch infolge des hohen Frauenanteils unter den Mitarbeitern bei steigender Raucherprävalenz von Frauen in der Bevölkerung (16). Gegenüber früheren Erhebungen aus Deutschland und anderen europäischen Ländern zeigt sich in der vorliegenden Studie ein niedriger Anteil rauchender Ärzte, hingegen ein sehr hoher Anteil von Rauchern innerhalb des Krankenpflegepersonals. Insgesamt finden sich bei einem Vergleich mit internationalen Erhebungen der letzten Jahre nur in Spanien (5) höhere Raucherprävalenzen bei Klinikpersonal, in allen anderen Staaten jedoch niedrigere klinikinterne Raucherraten $(12,14,22,24,33,36)$. Die vorliegenden Daten weisen somit auf die absolute Dringlichkeit der Förderung von Raucherentwöhnungen unter Klinikmitarbeitern in Deutschland.

Durch die nachfolgende Intervention waren nach 3 Monaten $38 \%$ und nach 6 Monaten 30\% der teilnehmenden rauchenden Mitarbeiter dauerhaft abstinent, hierbei fand sich ein (nicht signifikant) höherer Prozentsatz in Gruppe B. Somit konnte durch die gemeinschaftliche Aktion bei nicht selektierten gesunden Rauchern eine vergleichsweise hohe Abstinenzrate erzielt werden. Nach Daten einer systematisierten Übersicht ergeben sich für Interventionsprogramme mit NET plus intensiver psychologischer Betreuung durchschnittliche Abstinenzraten von 28,5\% (26,5-30,6\%) nach 6 Monaten (7). In den größeren kontrollierten Studien zur NET mit Kurzintervention lagen die Abstinenzraten bei etwa $20 \%$ gegenüber $10 \%$ unter Placebo (30). In der vorliegenden Untersuchung dürfte die höhere gesamte Abstinenzrate vor allem durch die intensive Motivationsförderung im Rahmen der gemeinschaftlichen innerbetrieblichen Aktion begründet sein. Während der gesamtem Laufzeit der Studie war das Thema Rauchen und „Rauchfreies Krankenhaus“ fester Bestandteil der innerbetrieblichen Kommunikation. Vergleichsweise hohe Abstinenzraten wurden auch bei ähnlichen klinikinternen Programmen in Australien und Dänemark berichtet $(1,15)$.

$\mathrm{Zu}$ allen Messzeitpunkten zeigten sich in Gruppe B mit Stressreduktion höhere, jedoch nicht signifikant erhöhte Abstinenzraten. Signifikante Unterschiede zugunsten der Stressreduktion fanden sich für die CO-Konzentration im Exspirium sowie für die Anzahl gerauchter Zigaretten bei rückfälligen Rauchern. Wenngleich die Reduktion des Rauchens die Prävalenz der tabakabhängigen Erkrankungen reduziert und oftmals der kontinuierlichen Abstinenz zeitlich vorausgeht, konnte damit kein kla- rer Nutzen des Stressreduktionsprogrammes aufgezeigt werden. Für die Einschätzung der Wirksamkeit der in der Studie durchgeführten „mindfulness based stress reduction“ (MBSR) ist jedoch auch die Adhärenz mit dem Programm zu berücksichtigen. Trotz des kostenfreien Angebotes und der Kursdurchführung innerhalb der Klinik nahmen nur 45\% der freiwillig in Gruppe B zugeordneten Teilnehmer regelmäßig am Kursprogramm teil und übten selbständig Entspannungsverfahren. Ein höherer Anteil der regelmäßig Übenden blieb hierbei gegenüber den Nicht-Übenden im Verlauf abstinent. In den amerikanischen Untersuchungen der Arbeitsgruppe um Kabat-Zinn konnten bei verschiedenen Krankheitsentitäten durchweg höhere Adhärenz-Raten erreicht werden (17-19). Die Wirksamkeit des verwendeten Stressreduktionsprogrammes sollte daher in Studien mit größeren Fallzahlen und Responderanalyse sowie stärkerer Berücksichtigung transkultureller Besonderheiten in der Schulungsmethode weiter überprüft werden.

Bei der Dateninterpretation sind die methodischen Limitierungen der Studie zu beachten. Die nicht-randomisierte Gruppenzuteilung wurde gewählt um den gemeinschaftlichen Charakter der Aktion „Rauchfreies Krankenhaus“ zu unterstützen und die externe Validität der Ergebnisse zu betonen. Durch das Fehlen der Randomisierung ist die Vergleichbarkeit der Gruppen im Sinne eines Selektionsbias grundsätzlich eingeschränkt. In der Regressionsanalyse waren die Abstinenzraten jedoch unabhängig von soziodemographischen Variablen sowie dem Fagerstrøm-Score und der Länge der Raucheranamnese.

Die mittlere Dauer des Rauchens von etwa 20 Jahren in der Gesamtpopulation und der überwiegend frühe Beginn der Rauchergewohnheit weisen darauf, dass die Nikotinabhängigkeit überwiegend berufsunabhängig bereits in Jugendjahren entstand und unterstreichen die Bedeutung der Primärprävention. Erwartungsgemäß kam es durch die Raucherentwöhnung zu einer signifikanten Gewichtszunahme, etwas geringer ausgeprägt in Gruppe B. Eine Gewichtszunahme war auch von mehr als der Hälfte der Teilnehmer als persönlich relevante Nebenwirkung des Raucher-Stopps befürchtet worden. Angst vor Gewichtszunahme ist vermutlich eine der Hauptbarrieren der Raucherentwöhnung (29).

\section{Fazit}

Zusammenfassend konnte in der Studie gezeigt werden, dass durch eine gemeinschaftliche innerbetriebliche Aktion mit ärztlich geführter medikamentöser NET eine vergleichsweise hohe Abstinenzrate bei Krankenhauspersonal zu erzielen ist. Es erscheint dringlich und machbar, die inakzeptabel hohe Raucherprävalenz an deutschen Kliniken durch entsprechende Aktivitäten sowie fördernde Strukturen, z.B. Etablierung eines „raucherbeauftragten Arztes“, zu senken. Der Stellenwert additiver Stressreduktionsprogramme sollte in größeren randomisierten Studien weiter evaluiert werden. 
Danksagung: Diese Studie wurde durch die freundliche Unterstützung der Firma Pharmacia, Erlangen, ermöglicht: personelle Unterstützung bei der Datenerhebung, Datenanalyse mittels eines unabhängigen statistischen Institutes sowie Vorhalten des Nikotinersatzes.

\section{Literatur}

${ }^{1}$ Backer V, Olsen AD, Enevoldsen HK, Munk P, Schioning H, Kann A, Mundt K, Agner T, Tonnessen H. Smoking intervention among hospital staff at the H.S. Bisperebjerg Hospital. Toward a non-smoking hospital - short term results. Ugeskr Laeger 1999; 161: 3663-3665

2 Balfour D, Benowitz N, Fagerstrøm T, Kunze M, Keil U. Diagnosis and treatment of nicotine dependence with emphasis on nicotine replacement therapy. Eur Heart J 2000; 21: 438-445

3 Batra A. Tabakabhängigkeit - Biologische und psychosoziale Entstehungsbedingungen und Therapiemöglichkeiten. Steinkopff, Darmstadt, 2000

${ }^{4}$ Berkel T, Boersma H, Roos-Hesselink J. Impact of smoking cessation and smoking interventions in patients with coronary heart disease. Eur Heart I 1999; 20: 1773-1782

${ }^{5}$ Carrion VF, Hernandez LJ, Plaza VP. Smoking in health care workers. Identification of related factors. Arch Bronconeumol 1998; 34: 379383

${ }^{6}$ CEASE Trial (Collaborative European Anti Smoking Evaluation). Higher dosage nicotine patches increase one-year smoking cessation rates: results from the European CEASE trial. Eur Respir J 1999; 13 238-247

7 Cinciripini PM, McClure JB. Smoking cessation: recent developments in behavioural and pharmacologic interventions. Oncology 1998; 12 : 249-256

8 Dornelas E, Sampson R, Gray JF, Waters D, Thompson PD. A randomized controlled trial of smoking cessation counseling after myocardial Infarction. Prevent Med 2000; 30: 261-269

9 Hajek P. Treatment for smokers. Addiction 1994; 89: 1543-1549

${ }^{10}$ Hajek P, West R, Foulds J. Randomized comparative trial of nicotine polacrilex, a transdermal patch, nasal spray, and an Inhaler. Arch Intern Med 1999; 159: 2033-2038

11 Haustein KO. Pharmacotherapy of nicotine dependence. Int J Clin Pharmacol 2000; 38: 273-290

12 Hay DR. Cigarette smoking by New Zealand doctors and nurses: results from the 1996 population consensus. N Z Med J 1998; 27: 102104

${ }^{13}$ Heatherton TF, Kozlowski LT, Frecker RC, Fagerstrøm KO. The Fagerstrøm test for nicotine dependence: a revision of the Fagerstrøm tolerance questionnaire. Br J Addict 1991; 86: 1119-1127

${ }^{14}$ Hughes AM, Rissel C. Smoking: rates and attitudes among nursing staff in central Sidney. Int J Nurs Pract 1999; 5: 147-154

15 Jones TE, Crocker H, Ruffin RE. Smoking habits and cessation programme in an australian teaching hospital. Aust N Z J Med 1998; 28: 446-452

${ }^{16}$ Junge B, Nagel M. Das Rauchverhalten in Deutschland. Gesundheitswesen 1999; 61: 121-125

17 Kabat-Zinn J, Chapman-Waldrop A. Compliance with an outpatient stress reduction program: rates and predictors of program completion. J Behav Med 1988; 11: 333-352

18 Kabat-Zinn J, Massion AO, Kristeller J, Peterson LG, Fletcher K, Pibert L, Linderking W, Santorelli SF. Effictiveness of a meditation-based stress reduction programm in the treatment of anxiety disorders. Am J Psychiatry 1992: 149: 936-943

19 Kabat-Zinn J, Miller J, Fletcher K. Three-year follow-up and clinical implications of a mindfulness meditation-based stress reduction intervention in the treatment of anxiety disorders. Gen Hosp Psychiatry 1995; 17: 192-200

20 Kornitzer M, Boutsen M, Dramaix M, Thijs J, Gustavsson G. Combined use of nicotine patch and gum in smoking cessation: a placebo-cotrolled trial. Prevent Med 1995; 24: 41-47

${ }^{21}$ Lancaster T, Stead LF. Self-help interventions for smoking cessation. (Cochrane Review). Oxford. Update Software, In : The Cochrane Library, Issue 1.2000

22 McKenna H, Slater P, Mc Cance T, Bunting B, Spiers A, McElwee G. Qualified nurses smoking prevalence: their reasons for smoking and desire to quit. J Adv Nurs 2001; 35: 769-775
${ }^{23}$ Murray CJL, Lopez AD. Mortality by cause for eight regions of the world: global burden of disease study. Lancet 1997; 349:1269-1276

24 Muzi G, dell Omo M, Crespi E, Madeo G, Monaco A, Curradi F, Diodati $\mathrm{R}$, Abbritti G. Smoking in the workplace. Study at a hospital in central italy. Med Lav 2001; 92: 54-60

${ }^{25} \mathrm{NN}$. A Clinical practice guideline for treating tobacco use and dependence: A US public health service report. The tobacco use and dependence clinical practice guideline panel. Staff and consortium representative. JAMA 2000; 283: 3244-3254

${ }^{26}$ NN . Medikamentöse Unterstützung bei der Raucherentwöhnung. Der Arzneimittelbrief 2000; 34: 25-29

27 Ohida T, Osaki Y, Kobayashi Y, Sekiyama M, Minowa M. Smoking prevalence of female nurses in the national hospitals of Japan. Tob Control 1999; 8: 192-195

28 Peto R, Lopez AD, Boreham J, Thun M, Heath JC. Mortality from smoking in developed countries 1950-2000. Indirect estimates from national vital statistics. Oxford: Oxford University press, 1994

29 Russ CR, Fonseca VP, Peterson AL, Blackman LR, Robbins AS. Weight gain as a barrier to smoking cessation among military personnel. Am J Health Promot 2001; 16: 79-84

30 Silagy C, Mant D, Fowler G, Lancaster T. Nicotine replacement therapy for smoking cessation (Cochrane Review). Oxford. Update Software, In: the Cochrane Library, Issue 1. 2000

31 Simon JA, Solkowitz SN, Carmody TP, Browner WS. Smoking cessation after surgery. A randomized trial. Arch Intern Med 1997; 157: 1371-1376

32 Troschke G. Gesundheitsberatung zur Tabakentwöhnung. Gustav Fischer, Jena, 1992

${ }^{33}$ Varsano S, Hevion G, Garenkin M. Smoking by an Israeli general hospital staff, and attitude to smoking in hospitals.:Are we in Israel ready to institute „smoke-free-hospitals?“ Harefuah 2000; 138: 335-340

${ }^{34}$ Weißbuch Lunge 2000, G. Thieme, Stuttgart, Deutsche Lungenstiftung, Deutsche Gesellschaft für Pneumologie. 2000

35 Weltgesundheitsorganisation. „Partnerschaftsprojekt Tabakabhängigkeit 1999-2001“ Deutsches Krebsforschungszentrum, Heidelberg, 1999

${ }^{36}$ Zanetti F, Gambi A, Bergamaschi A, Gentillini F, De Luca G, Monti C, Stampi S. Smoking habits, exposure to passive smoking and attitudes to a non-smoking policy among hospital staff. Public Health 1998; 112: $57-62$ 